\title{
Bracing for a resurgence of Covid in a 3rd Wave: Uttarakhand Saga
}

Debabrata Roy ${ }^{1}$, Richa Sinha², Deepshikha ${ }^{3}$

${ }^{1}$ Professor \& Head, Department of Community Medicine, Government Doon Medical College, Patel Nagar, Dehradun, Uttarakhand 248001; ${ }^{2}$ Assistant Professor, Department of Community Medicine, Government Doon Medical College, Patel Nagar, Dehradun, Uttarakhand 248001; ${ }^{3}$ Associate Professor, Department of Community Medicine, Himalayan Institute of Medical Sciences, Swami Rama Himalayan University, Dehradun, Uttarakhand 248016

\section{Corresponding Author}

Dr Richa Sinha, Assistant Professor, Department of Community Medicine, Government Doon Medical College, Patel Nagar, Dehradun, Uttarakhand 248001

E Mail ID: richaparmarth@gmail.com

\section{Citation}

Roy D, Sinha R, Deepshikha. Bracing for a resurgence of Covid in a 3rd Wave: Uttarakhand Saga. Indian J Comm Health. 2021;33(4):547-548. https://doi.org/10.47203/IJCH.2021.v33i04.001

Source of Funding: Nil Conflict of Interest: None declared

\section{Article Cycle}

Received: 10/11/2021; Revision: 05/12/2021; Accepted: 15/12/2021; Published: 31/12/2021

This work is licensed under a Creative Commons Attribution 4.0 International License.

Evidently, the resurgence of COVID in a 2nd wave happened due largely to 'Risk Compensation' by the community, exacerbated by extreme risk behavior of the community. 'Risk compensation' or 'Peltzman effect', where there is an attitudinal shift underestimating the risk and subsequent complacency in observing due measures is almost an axiomatic phenomenon and this was being predicted in case of COVID too(1). However the scale, extent and intensity of the re-surgence (second wave) which was exponential \& 'asymptotic' was unwarranted, also unprecedented. That this re-surgence was a predictable phenomenon and needed pro-active \& pragmatic strategizing/ decision making (which would affect community behavior vis a vis religious congregation and or other public events abetting gathering of crowd etc) was not unknown.

It was argued that a $3^{\text {rd }}$ wave of COVID re-surgence, if at all happened, might only be possible if we failed to apply 'lessons learnt 'so far in terms of primordial/ primary prevention (sensitizing \& building community capacity to continue to adopt COVID appropriate practices and without any relent) measures so that, irrespective of any further variant/s appearing, we did our best not to allow the virus to make us its host. This holds good on principle and by all means even now, that Variants of Concern $(\mathrm{VoC})$, among these essentially, B. 1.1.529, otherwise named Omicron, has appeared on the scene, its mutagenicity already showing pandemic potential.

Meanwhile a few significant attributes of \& during the second re-surgence were / are:

a) Appearance of Genomic variants of SARS - COVID 2 (including Omicron, Delta micron) b) Epidemic curve for second re-surgence that had been at fulminant community transmission mode till about the early second week of May, 2021 (daily case incidence reached its 'Peak' virtually around May 7/ 8,2021 , then plateaued till about May 12, 2021) had been consistently declining since.

c) Proportional mortality rate / case fatality rate in the state did not remarkably change during the resurgence (the range being $2.2 \%$ )

d) As many as $73 \%$ of population samples from the state whose genomes were sent for genomic sequencing with a view to see the extent, type of mutation etc, showed sequence of the original SARS COVID 2; UK variants were $12 \%$ and others $15 \%$ (285 samples sequenced out of 822; at the time of analysis the test results of the rest were awaited) (2)

e) The second wave affected virtually all age groups and gender and in manner unlike in the first one.

f) A mass prophylaxis regimen including Ivermectin had been instituted during May, 2021 by Government of Uttarakhand and evidences exist associating Ivermectin administration with the decline of case growth rate i.e rate of descent of the curve by $81 \%$ during $3^{\text {rd }}$ week of administration in Uttarakhand.

g) Immunization or specific protection against SARS COVID-2 (Covishield, Covaxin and Sputnik), started on \& from $16^{\text {th }}$ January, ' 21 , is ongoing in the State.(3)

h) It is perhaps not convincing to believe that children have been less exposed so far, in that, a COVID positive parent / individual in a household may be assumed to have surely come into contact with the children too. 
At the circumstances, it may not be farfetched to presume that we as a 'herd' might have attained to a collective immune competence synonymous with a 'community shield' which was getting reinforced by the day with ongoing vaccination (seroprevalence studies across India revealed a $60 \%$ or more of the population of most of the States showing evidence of antibodies against SARS CoV2 implying that a community shield might have been already established)(4)

The scenario appears to be changing as newer variants of concern including Omicron continue to spread as feared what with the much faster transmissibility, much less 'doubling time', reported 'escaping' of earlier immunecompetence either by infection with the delta variant or by vaccination, its lower virulence notwithstanding. We may indeed then brace for another probable resurge of the epidemic with pandemic potential in a densely populated country like India and abetted by a complacent community, at the moment largely in a state of 'risk compensation' as evidenced earlier.

Interestingly, In India the epidemic curve due to the delta variants had been consistently touching base till barely two weeks ago and now, the surge across the country is exponential! What, after all, is / are driving this resurgence in our country? Is it merely Omicron (as is being given to understand)? Well, paradoxically \& interestingly, a very miniscule proportion of resurgent cases across India is due to Omicron (as media says)- just about 4000 plus cases, gnomically sequenced as Omicron pan India and merely 08 cases in Uttarakhand, at the time of going to the press.

So then, what may have been the predisposing and precipitating factors of this essentially delta variant resurgence either across Europe or in India? May it be presumed that immune competence of the community did wane with time and the 'community shield' thus became porous over time? or the delta variant underwent sub type mutations other than merely Omicron?

It has been apt strategizing by the Government to have decided upon introducing boosters and bringing in virtually all vulnerable \& age groups under the ambit of vaccination with the advent of the new year.

At the circumstances, in view of an impending resurgence of Covid disease in a third wave, it is pertinent that 'lessons learnt 'so far in terms of primordial/ primary prevention (sensitizing \& building community capacity to continue to adopt COVID appropriate practices and without any relent) have to be the primary guiding principle in management of the situation.

Pragmatic planning shall be necessary in deciding on measures of prevention and control taking into account all affairs of life \& livelihood, namely in terms of staggered \& graded responses. Importantly, restrictions have remained at best at lock down level 1 at badly hit locales across the world despite holiday seasons \& festivities. Priority may be accorded to restricting public gatherings, electioneering rallies -emphasizing media mode of campaign, religious congregations, public celebrations etc.

Management of cases in isolation at home acquires renewed importance at the present circumstances and have to be effectively strengthened with capacity building, sensitization of both providers \& the community (as per revised guidelines by the government for mild/ asymptomatic Covid 19 cases)

Further, to this end, other key measures i.e. reinforcement of existing Health programmes / initiatives at State or National level, essentially to address Reproductive \& Child health $(\mathrm{RCH})$ at different levels of Primary Health Care with active community participation is a felt need we must not underestimate.

\section{References}

1. Peltzman $S$. The effects of automobile safety regulation. J Polit Econ $1975 ; 83: 677-725$.

2. Indian SARS-CoV-2 Genomics Consortium. 25th June 2021. Ministry of Health \& Family Welfare, GOI.

3. Ministry of Health and Family Welfare, "Cumulative Covid Vaccination Report 2nd June 2021".

4. Technical paper COVID 19 ICMR - Serological Surveillance Report Round 4Department of Health \& Family Welfare. 